\title{
Differential effects of experimentally induced anxiety and fear on pain: the role of anxiety sensitivity
} This article was published in the following Dove Press journal:
Journal of Pain Research

Background: Anxiety has been associated with both increased and decreased pain perception. Rhudy and Meagher (2000) showed that pain sensitivity is enhanced by anxiety (anticipation of shocks), but diminished by fear (confrontation with shocks). A problem of this approach is the confounding of emotional and attentional effects: Administered shocks (fear induction) divert attention away from pain, which might account for lower pain in this condition. Moreover, heterogeneous findings in the past might be due to inter-individual differences in the proneness to react to anxiety and fear such as ones anxiety sensitivity (AS) level.

Objectives: Our aim was to clarify the association between anxiety, fear and pain. We used the NPU paradigm for inducing these emotions and recording pain sensitivity at once with one stimulus to prevent interference by distraction. We assumed that anxiety and fear affect pain differently. Moreover, we hypothesized that subjects with clinically relevant (high) AS (H-AS group) show enhanced pain perception in contrast to low AS subjects (L-AS group). Method: Forty healthy subjects (female: $\mathrm{N}=20$; age $\mathrm{M}=23.53$ years) participated and $\mathrm{H}-\mathrm{AS}$ or L-AS status was determined by clinically discriminating cut-off scores of the Anxiety Sensitivity Index-3 (ASI-III). Emotions were induced by the application of unpredictable (anxiety) and predictable (fear) electric stimuli. Pain ratings of electric stimuli were compared between the conditions. Startle reflex and anxiety ratings were recorded.

Results: Results showed no general effects of anxiety and fear on pain perception. However, anxiety enhanced pain sensitivity in H-AS subjects, whereas fear did not affect pain sensitivity. In L-AS subjects no effects on pain perception were found.

Conclusion: Results revealed that anxiety, not fear, enhanced pain perception but only in subjects with clinically relevant AS levels. This indicates that subclinical AS levels are sufficient to increase pain sensitivity, in uncertain situations.

Keywords: anxiety sensitivity index-3, enhanced pain sensitivity, attention effects, electric stimuli

\section{Introduction}

Studies have revealed no one-to-one relationship between the intensity of nociceptive stimuli and pain perception, rather, this relationship is modulated by various factors, psychological factors being among them. ${ }^{1-5}$ A considerable focus lies on the investigation of the modulating effect of anxiety and fear on pain, because these emotions are playing a crucial role in the development and maintenance of chronic pain. $^{6-8}$ Of course, anxiety and fear also seem to influence acute and experimental pain. ${ }^{1,9-12}$ Anxiety has been associated with both increased and decreased pain
Correspondence: Silvia Metzger Physiological Psychology, Otto-FriedrichUniversity of Bamberg, Markusplatz 3, Bamberg D-96045, Germany

Tel +49 95I 8632249

Email silvia.metzger@uni-bamberg.de 
perception. ${ }^{11,13,14}$ These controversial findings might be due to a missing differentiation between anxiety and fear in experimental studies, although anxiety and fear are two different psychological states. ${ }^{1,11,15-17}$ Fear arises as a direct response to an aversive stimulus, is accompanied by automatic arousal and activates the "fight or flight" response (eg, fight against or run away from a predator). To ensure the survival of the organism in the face of an acute threat, pain is inhibited (eg, the perception of painful injury might be suppressed to allow for fighting or taking flight). In contrast, anxiety is a longer lasting diffuse feeling related to the uncertain anticipation of threat and can lead to hypervigilance. ${ }^{17,18}$ Hypervigilance results in scanning of the environment and enhances sensitivity to painful events. ${ }^{19,20}$ There are several methods to induce anxiety and fear in experimental studies, for example, the presentation of emotional pictures or movie scenes. ${ }^{16,21}$ Furthermore, fear can be induced by a cue that was paired with aversive stimuli (eg, electric stimuli). Thereby, the cue made the electric stimuli predictable. ${ }^{22,23}$ Anxiety instead is often elicited by triggering the anticipation of unpredictable aversive stimuli. ${ }^{11,15,22,23}$

The seminal study of Rhudy and Meagher (2000) showed that anxiety and fear have divergent effects on pain, whereby anxiety increases pain sensitivity (anxietyinduced hyperalgesia) and fear decreases it (fear-induced analgesia). Anxiety and fear were induced by the actual application (fear) or the mere announcement (anxiety) of electric shocks at the index finger of the dominant hand. Heat pain thresholds were assessed at the index finger of the other hand to identify the influence of anxiety and fear on pain. One concern within this approach is that emotioninducing stimuli (electric shocks) - due to their salience draw spatial attention away from the site of the application of painful stimuli (heat stimuli). ${ }^{24,25}$ Distraction itself can reduce pain perception without the involvement of emotions.9,26,27 Thus, a distraction effect might account for the lower pain sensitivity observed in the fear condition, where shocks were truly applied and not only announced as in the anxiety condition. ${ }^{11}$

To resolve this methodological problem, a paradigm is required in which interference by distraction is ruled out. The "Neutral Predictable Unpredictable threat paradigm" (NPU paradigm), ${ }^{22}$ which is one of the gold standards to investigate anxiety and fear, might be a promising candidate. In the NPU paradigm, anxiety and fear are induced by the application of unpredictable (anxiety) and predictable (fear) electric stimuli. Startle reflex and anxiety ratings are recorded to check for a successful anxiety and fear induction. ${ }^{22}$ The advantage of this paradigm is the possibility of using only one stimulus (electric stimuli) for inducing emotional states (anxiety and fear) and measuring pain sensitivity at the same time.

The major aim of our study was to investigate the influence of anxiety and fear on pain perception with the NPU paradigm. Additionally, we were interested in the influence of anxiety sensitivity (AS) on pain perception, in interaction with anxiety and fear, as heterogeneous findings in the past might be due to inter-individual differences in the proneness to react to anxiety and fear. Subjects with high AS tend to interpret bodily sensations (eg, an enhanced heart rate) as predicting harm and seem to be particularly vulnerable to unpredictable threat, which might lead to a heightened level of anxiety in uncertain situations. ${ }^{28-30}$ Studies have revealed that high AS which is a risk factor for the development of anxiety disorders also enhances acute pain and increases the likelihood for chronic pain. ${ }^{31-33}$ AS and pain perception may be mediated by other factors like state anxiety, fear of pain or pain catastrophizing. ${ }^{7,34-36}$

We hypothesized that subjects perceive the painfulness of the electric stimuli differently in the anxiety condition as compared to the fear condition. Furthermore, we assumed that the effects of anxiety and fear on pain perception depend on the individual level of AS assessed by the Anxiety Sensitivity Index-3 (ASI-III). ${ }^{37,38}$ In our study, we used AS in a categorical fashion. Allan and colleagues (2014) showed that subjects with high AS (sum score $>23$ ) have an increased risk for the development of anxiety disorders, in contrast to subjects with low AS scores (sum score $<23$ ). ${ }^{39}$ Therefore, we dichotomized our study cohort into two groups; a high AS (H-AS) group, that included subjects with an increased likelihood for developing clinically relevant anxiety states (sum score $>23$ ) and a low AS (L-AS) group containing subjects with low AS (sum score $<23$ ). We expected that subjects with clinically relevant AS (H-AS group) show enhanced pain perception in contrast to low AS subjects (L-AS group).

\section{Methods}

\section{Subjects}

Forty healthy, pain-free students (female: $\mathrm{N}=20$ ) between the ages of 20 and 28 years (mean age: 23.53 years; $\mathrm{SD}=2.2$ ) participated in this study. Participants were recruited by advertisements at the University of Bamberg and H-AS or L-AS status was determined by cut-off scores 
of the ASI III. ${ }^{37,38}$ We used a student sample, because we know from previous studies that we find enough subjects for both groups (H-AS and L-AS) in this population. Prior to the experiment, which was carried out in a laboratory of the University of Bamberg, a phone interview was conducted to exclude participants with acute and chronic mental or physical diseases. On the experimental day, subjects were requested to refrain from drinking coffee and smokers were requested to stop smoking $1 \mathrm{hr}$ before start of the experiment to prevent influences on arousal with consequences on anxiety, fear and pain. All subjects provided written informed consent and received monetary compensation for their participation or course credits (psychology students). The ethics committee of the University of Bamberg approved the experimental procedure.

\section{Procedure}

During the session, subjects were sitting in a comfortable chair in front of a computer display. After having given their informed consent, subjects were prepared for the experimental procedure. For the electrical stimulation, two electrodes were attached to the subjects' forearm. Additionally, for the startle reflex measurement, three electrodes were attached around the subjects' left eye (for details see paragraph "Startle reflex" below). For the electrical stimulation, individual levels of stimulation intensity were determined (between $1 \mathrm{~mA}$ and $5 \mathrm{~mA}$ ) according to the protocol of Grillon and colleagues. ${ }^{22}$ The target value was an intensity of the electric stimuli which subjects perceived as "quite unpleasant" (for details see paragraph "Electric stimulation: apparatus and intensity" below). After that, subjects completed the German Version of the ASI-III. ${ }^{37,38}$ To elicit the startle reflex, we applied brief acoustic stimuli. For this purpose, subjects put on headphones (for details, see paragraph "Startle probes" below). Subsequently, the experiment started. An overview of the experimental procedure is shown in Figure 1.

Participants completed two blocks (block 1 and block 2) of the NPU paradigm. Each block consisted of three conditions: $\mathrm{N}$ (no electric stimuli), P (predictable electric stimuli, used to induce fear) and $U$ (unpredictable electric stimuli, used to induce anxiety). The two blocks only differed regarding the sequence of the three conditions (sequence in block 1 : $\mathrm{P}, \mathrm{N}, \mathrm{U}$; sequence in block 2: U, N, P). There was a 5-min break between both blocks. As high as $50 \%$ of the subjects started with block 1 and 50\% started with block 2 to control for order effects. After each condition, subjects rated their perceived anxiety. Additionally, they rated painfulness, intensity and threat of the electric stimuli after condition $\mathrm{P}$ and $U$ by the use of a numerical rating scale. The whole NPU paradigm lasted about 50 mins (without preparation and without completing the questionnaire).

\section{Anxiety and fear induction}

In each condition of the NPU paradigm, ${ }^{22}$ a specific visual cue (P: red square, N: green circle, $\mathrm{U}$ : blue triangle) was repeatedly presented for $8 \mathrm{~s}$ with an inter-stimulus interval (ISI) between 30 and $35 \mathrm{~s}$. In the condition P, electric stimuli were administered only in the presence, but not in the absence of the visual cue (predictable condition). In the condition $\mathrm{N}$, no electric stimuli were delivered (safe condition). In the condition $U$, electric stimuli were possible at any time, ie, both in the presence and the absence of the visual cue (unpredictable condition). This results in two options for each condition: cue present $\left(\mathrm{P}_{\text {cue }}, \mathrm{N}_{\text {cue }}, \mathrm{U}_{\text {cue }}\right.$ or cue absent ( $\left.\mathrm{P}_{\text {nocue }}, \mathrm{N}_{\text {nocue }}, \mathrm{U}_{\text {nocue }}\right)$. An instruction on the computer screen informed subjects about the present condition (in condition $\mathrm{P}$ "shock only during red square", in condition $\mathrm{N}$ "no shock" and in condition U "shock at any time").

To check whether emotional responses were successfully induced, the startle (blink) reflex, which is an automatic reaction to sudden aversive events like loud tones $^{23,40}$ was measured and anxiety ratings were recorded in each condition. It has consistently been shown that threatening stimuli potentiate the amplitude of the blink reflex. $^{22,23,40,41}$ A successful induction of anxiety and fear can be inferred from a specific pattern in startle amplitudes within and across conditions. Typically, in the condition P, mean startle amplitudes are higher in the presence than in the absence of the visual cue $\left(\mathrm{P}_{\text {cue }}>\mathrm{P}_{\text {nocue }}\right)$, representing the "fear-potentiated startle". Mean startle amplitudes during the absence of the cue in the condition $U$ are higher than during the condition $\mathrm{N}\left(\mathrm{U}_{\text {nocue }}>\mathrm{N}_{\text {nocue }}\right)$, representing the "anxiety-potentiated startle". For anxiety ratings, the same pattern is expected. ${ }^{22,23}$

\section{Electric stimulation: apparatus and intensity}

After the skin abrasion, two 4-mm stimulating electrodes $(\mathrm{Ag} / \mathrm{AgCl})$ were attached with a distance of $3 \mathrm{~cm}$ from each other at the midline of the volar central forearm of the non-dominant hand. A constant-current stimulator (Pulsar 6i) delivered the electric stimuli, which were trains of 50 square wave pulses with a total duration of $246 \mathrm{~ms}$. Each pulse had a duration of $1 \mathrm{~ms}$ with an interval of $4 \mathrm{~ms}$ between pulses. Electric pulses are shown in Figure 2. To make the NPU paradigm usable in a pain experiment, it was necessary to 


\section{Preparation}

Fixing electrodes on M. orbicularis oculi and on the volar distal forearm of the non-dominant hand, predetermination of stimulus intensity
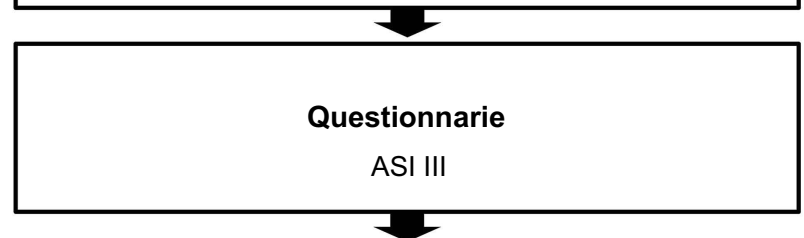

NPU

Paradigm

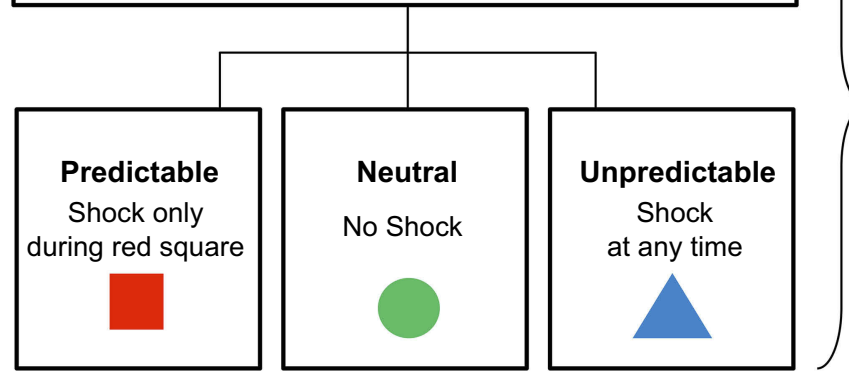

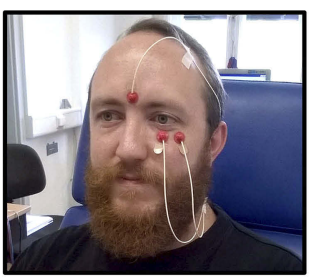

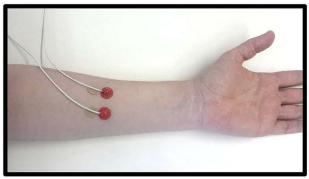

Startle Reflex

\&

Anxiety Rating

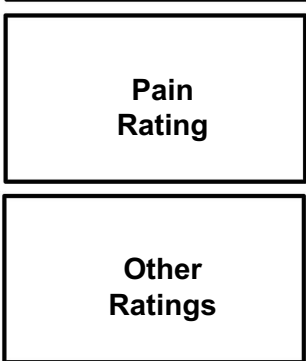

Figure I Overview of the experimental procedure.

Abbreviations: NPU paradigm, neutral predictable unpredictable threat paradigm; ASI III, Anxiety Sensitivity Index-3.

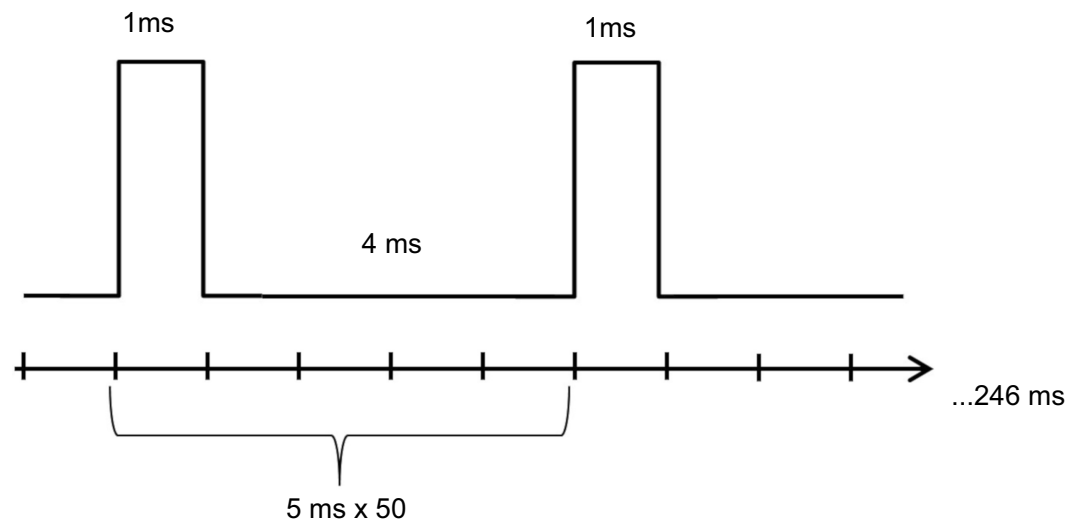

Figure 2 One single square pulse.

Abbreviation: ms, milliseconds.

record responses to a sufficient number of pain stimuli. Therefore, we increased the number of the electric stimuli from 2 to 3 in condition $P$ and $U$ and prolonged each condition (original NPU: $120 \mathrm{~s}$ per condition; adapted NPU: $360 \mathrm{~s}$ per condition). In condition $\mathrm{P}$, all stimuli were delivered in the presence of the cue $\left(\mathrm{P}_{\text {cue }}\right)$. In condition $\mathrm{U}$, one electric stimulus was administered in the presence of the cue $\left(\mathrm{U}_{\text {cue }}\right)$ and two stimuli in the absence of the cue $\left(\mathrm{U}_{\text {nocue }}\right)$.
Prior to the start of the NPU protocol, the individual level of stimulation intensity was determined in order to tailor stimulation to the subject's pain sensitivity. Subjects were asked to verbally rate each electric stimulus on a 5point numerical rating scale ("1 - barely noticeable", "2 clearly noticeable but not unpleasant", "3 - barely unpleasant", "4 - quite unpleasant" to "5 - very unpleasant"). The first electric stimulus was delivered with an intensity of 0.5 
$\mathrm{mA}$. Intensity was increased in steps of $0.5 \mathrm{~mA}$ until subjects rated the electric stimulus with "4 - quite unpleasant" or until the maximum of $5 \mathrm{~mA}$ was reached. This procedure was repeated one more time, resulting in two runs. Later in the NPU paradigm, we used the intensity corresponding to a rating of " 4 - quite unpleasant" in the second run.

\section{Startle probes}

To elicit the startle reflex, we presented short noise bursts (white noise, $105 \mathrm{db}, 50 \mathrm{~ms}$ ) binaurally over headphones above constant noise (68 db) for background masking. Eighteen noise bursts (startle probes) were presented in each condition (P, N and $\mathrm{U})$. Presentation occurred with an inter-stimulus interval (ISI) ranging randomly from 19 to 21 $\mathrm{s}$, with no tones during rating periods (after each condition). Nine startle probes were administered in the presence $\left(\mathrm{P}_{\text {cue }}\right.$, $\left.\mathrm{N}_{\text {cue }}, \mathrm{U}_{\text {cue }}\right)$ and nine in the absence of the visual cue $\left(\mathrm{P}_{\text {nocue }}\right.$, $\mathrm{N}_{\text {nocue }}, \mathrm{U}_{\text {nocue }}$ ). The electric stimuli and the startle probes were never presented simultaneously.

Each block (block 1 and block 2) was preceded by a startle habituation phase. The habituation phase consisted of nine acoustic startle stimuli at the beginning of block 1 and of four acoustic startle stimuli at the beginning of block 2. The startle reflex shows a strong habituation especially within the first few trials. ${ }^{22}$ This procedure prevented the occurrence of habituation during the test phase, which might influence the results. Overall, there were 121 startle probes (18 per condition resulting in 54 per block, 108 overall for the two blocks and 13 for saturation of habituation).

\section{Manipulation check Startle reflex}

The startle reflex was measured by recording surface EMG activity from the $M$. orbicularis oculi beneath the left eye (recording device: SIGMA Plpro/Type Databox DB 36; SIGMA Medizin-Technik GmbH, Gelenau, Germany). Two $4 \mathrm{~mm}$ recording electrodes $(\mathrm{Ag} / \mathrm{AgCl})$ were placed on the skin surface overlaying the orbicularis oculi muscle. A signal ground electrode was attached at the forehead. The recording bandwidth of the EMG signal was between $0.2 \mathrm{~Hz}$ and $300 \mathrm{~Hz}$; input resistance was above $20 \mathrm{~m} \Omega$. The signal was sampled at a rate of $512 \mathrm{~Hz}$. To allow for event-related analysis of signals, triggers were automatically set to mark the onset of the startle noise. After recording, data were analyzed offline with "Vision Analyzer" (Brain Products, Munich, Germany). Analysis included filtering of the signal $(50 \mathrm{~Hz}$ notch filter, $20 \mathrm{~Hz}$ high-pass filter and $256 \mathrm{~Hz}$ low-pass filter) as well as rectifying and integrating the signal. This integration procedure was executed over a time interval from 0 to $250 \mathrm{~ms}$ after noise burst onset. Responses without peaks between 30 and $100 \mathrm{~ms}$ after burst onset were excluded. Furthermore, trials with responses that did not fit the typical shape of a startle response were not considered for further analysis. Startle amplitudes were defined as voltage difference between the average baseline and voltage peak within a time frame of 30-100 ms after startle noise onset. Our measures and analyses were based on recommendations by Blumenthal et $\mathrm{al}^{42}$ and were successfully applied in several previous studies. ${ }^{43-45}$ Mean values of startle amplitudes were calculated for each condition in the presence and the absence of cues $\left(\mathrm{P}_{\text {cue }}, \mathrm{P}_{\text {nocue }}, \mathrm{N}_{\text {cue, }}\right.$ $\mathrm{N}_{\text {nocue }}, \mathrm{U}_{\text {cue }}, \mathrm{U}_{\text {nocue }}$ ).

\section{Anxiety ratings}

After each condition ( $\mathrm{P}, \mathrm{N}$ and $\mathrm{U})$, subjects rated their anxiety in the presence and in the absence of the cue on a 10-point numerical rating scale, ranging from " 1 - not anxious" to "10 - extremely anxious".

\section{Pain perception}

\section{Painfulness of electric stimuli}

After the conditions $\mathrm{P}$ and $\mathrm{U}$, subjects rated the painfulness of the electric stimuli on a 10-point numerical rating scale, ranging from " 1 - not at all painful" to " 10 - very painful".

\section{Other ratings} Intensity of electric stimuli

After the conditions $\mathrm{P}$ and $\mathrm{U}$, subjects rated the intensity of the electric stimuli on a 10-point numerical rating scale, ranging from "1 - very mild" to "10 - high".

\section{Threat of electric stimuli}

After the conditions $\mathrm{P}$ and $\mathrm{U}$, subjects rated the threat of the electric stimuli on a 10 -point numerical rating scale, ranging from "1 - low" to "10 - high".

\section{Assessment of anxiety sensitivity Anxiety Sensitivity Index-3 (ASI III)}

The ASI III ${ }^{37,38}$ was developed to measure the sensitivity for anxiety symptoms related to normal signs of sympathetic activation like a fast heart rate, trembling or cold hands. People with high scores in ASI III $^{37,38}$ tend to interpret these symptoms as threatening. The questionnaire 
contains 18 items and is divided into 3 subscales: physical concerns, cognitive concerns and social concerns. Items are rated on a 5-point numerical rating scale, ranging from "0 - disagree" to "4 - agree". For further analyses, we used the combined sum score (range 0-72). Allan and colleagues (2014) showed that subjects with high AS (sum score $>23$ ) have an increased risk for the development of anxiety disorders in contrast to subjects with lower AS scores (sum score $<23$ ). ${ }^{39}$ Therefore, we dichotomized our study cohort into a high AS (H-AS) group, that included subjects with clinically relevant anxiety scores (sum score $>23$ ) and a low AS (L-AS) group containing subjects with low AS (sum score $<23$ ). The ASI III ${ }^{37,38}$ showed good internal consistency (Cronbach's $\alpha=0.86$ ).

\section{Statistical analyses}

Two participants were excluded from further data analyses because their startle amplitudes deviated from the sample means by more than 2 SD. Therefore, all analyses were calculated with $\mathrm{N}=38$.

As manipulation check, we compared startle amplitudes and subjective anxiety ratings across conditions and cues by using a 3 (condition: P, N, U) x 2 (cue: presence or absence of the cue) repeated measures ANOVA and post-hoc paired-t-tests.

In order to test the differential effects of anxiety and fear on pain, we calculated paired sample $t$-tests and compared pain ratings of the electric stimuli between anxiety (condition $U$ ) and fear (condition $\mathrm{P}$ ). Furthermore, in order to explore the influence of the level of AS on pain ratings in the $\mathrm{P}$ and $\mathrm{U}$ conditions, we computed a repeated measures ANOVA with AS group (H-AS/L-AS) as between-subject factor, and condition (P, $\mathrm{U})$ as within-subject factor. As necessary, we used posthoc independent $t$-tests with AS group (H-AS/L-AS) as grouping variable.

Additionally, we used paired sample $t$-tests to compare the other ratings of the electric stimuli (intensity and threat of electric stimuli) between anxiety (condition $U$ ) and fear (condition P). To investigate the influence of AS on threat and intensity ratings in the $\mathrm{P}$ and $\mathrm{U}$ conditions, we computed two further repeated measures ANOVAs with AS group (H-AS/L-AS) as between-subject factor and condition $(\mathrm{P}, \mathrm{U})$ as within-subject factor.

We calculated difference scores between the conditions $\mathrm{P}_{\text {cue }}$ and $\mathrm{P}_{\text {nocue }}$ and between the conditions $\mathrm{U}_{\text {nocue }}$ and $\mathrm{N}_{\text {nocue }}$ for both anxiety ratings and startle amplitude to obtain indicators for the size of fear responses/fear-potentiated startle
$\left(\mathrm{P}_{\text {cue }}-\mathrm{P}_{\text {nocue }}\right)$ and anxiety responses/anxiety-potentiated startle $\left(\mathrm{U}_{\text {nocue }}-\mathrm{N}_{\text {nocue }}\right)$. We compared fear responses/fear-potentiated startle and anxiety responses/anxiety-potentiated startle between H-AS and L-AS subjects by paired sample $t$-tests.

Because ratings and startle amplitudes were not normally distributed, data were square root transformed. When the assumption of sphericity was nevertheless violated, Greenhouse-Geisser correction was used. For Ftests, partial eta squared $\left(\eta^{2}\right)(0.01$ : small effect; 0.06 : medium effect; 0.14: large effect) is reported as an estimate of effect size. Significance level was set $\alpha=5 \%$. According to our hypothesis, we investigated the influence of AS on pain ratings with one-tailed tests, whereas all other analyses were calculated two-tailed. To analyze data SPSS was used (IBM SPSS Statistics Version 22.0, Armonk, NY: IBM Corp).

\section{Results}

\section{Sample characteristic}

We analyzed data of 38 participants (19 female). Mean age of the whole sample was 23.63 years $(\mathrm{SD}=2.16)$ (female: $\mathrm{M}=23.84$, $\mathrm{SD}=1.86$; male: $\mathrm{M}=23.42, \mathrm{SD}=2.46$ ). Mean value of the ASI III $^{37,38}$ sum score was 25.74 $(\mathrm{SD}=12.00)$, which means that the majority of our sample was relatively high anxiety sensitive. ${ }^{39}$ After generating groups with low and high AS subjects by using the cut-off scores of Allan, ${ }^{39}$ the mean score for L-AS subjects $(n=17)$ was $15.00(\mathrm{SD}=5.65)$ and the mean score for H-AS subjects $(n=21)$ was $34.43(\mathrm{SD}=7.98)$.

\section{Manipulation check Startle amplitudes}

Results showed that anxiety and fear were successfully induced, considering the following criteria (see Figure $3 \mathrm{~A}$ ). In the condition $\mathrm{P}$, mean startle amplitudes should be higher in the presence than in the absence of the visual cue $\left(\mathrm{P}_{\text {cue }}>\mathrm{P}_{\text {nocue }}\right)$, representing the "fearpotentiated startle". Mean startle amplitudes during the absence of the cue in the condition $U$ should be higher than during the condition $\mathrm{N}\left(\mathrm{U}_{\text {nocue }}>\mathrm{N}_{\text {nocue }}\right)$, representing the "anxiety-potentiated startle". We found a main effect of condition (P, N, and $\mathrm{U})(F(1.73,63.96)=19.39$, $\left.p<0.001, \eta^{2}=0.34\right)$ on the startle amplitude, a main effect of cue (presence or absence of the cue) $(F(1,37)$ $\left.=50.04, p<0.001, \eta^{2}=0.58\right)$ and a two-way interaction of condition and cue $(F(1.62,59.86)=15.31, \quad p<0.001$, 

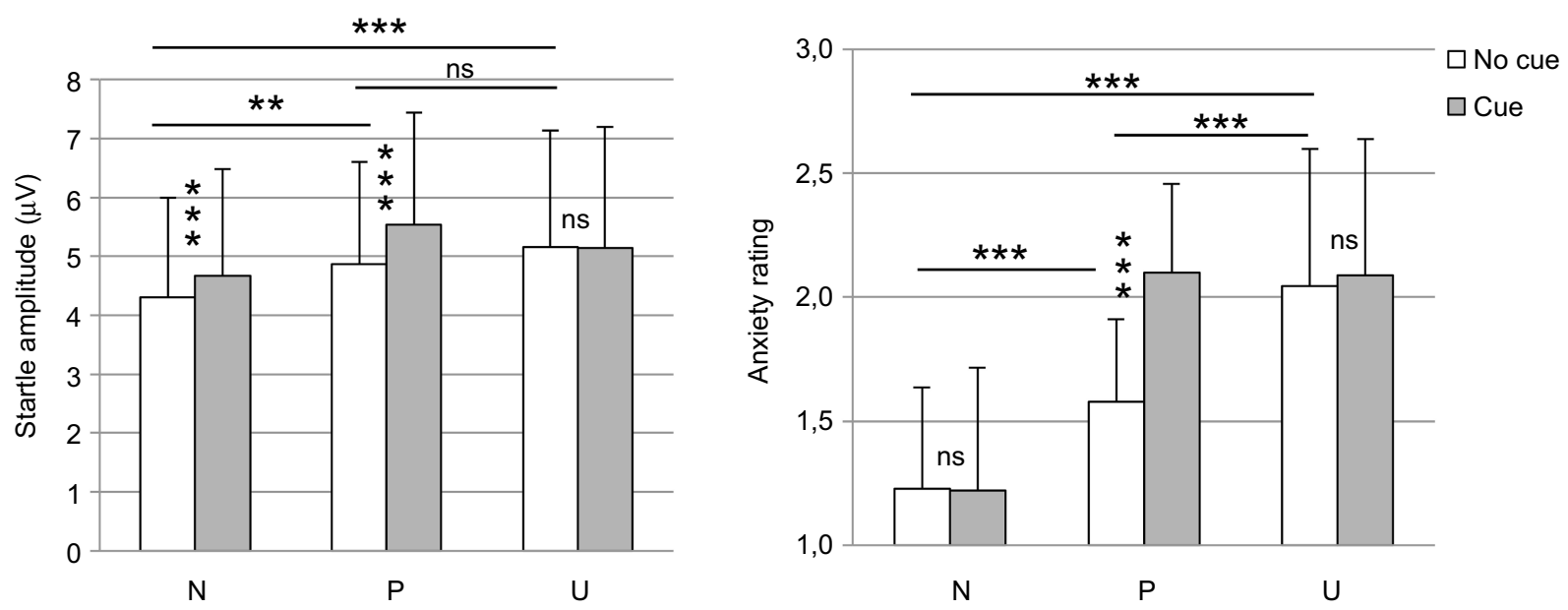

Figure 3 (A-B) Mean of startle reflex amplitudes (left) and anxiety ratings (right) in the three conditions $\mathrm{N}, \mathrm{P}, \mathrm{U} ; *^{* *} p<0.00 \mathrm{I}$; $* * p<0.0 \mathrm{I} ;{ }^{*} p<0.05$; bars represent standard deviation; data were square root transformed.

Abbreviations: $N$, neutral; $P$, predictable; $U$, unpredictable; ns, not significant.

$\left.\eta^{2}=0.29\right)$. Post hoc $t$-tests confirmed that both fearpotentiated startle $\left(\mathrm{P}_{\text {cue }}>\mathrm{P}_{\text {nocue }} ; t(37)=5.78, p<0.001\right)$ and anxiety-potentiated startle $\left(\mathrm{U}_{\text {nocue }}>\mathrm{N}_{\text {nocue }} ; t(37)\right.$ $=6.58, p<0.001)$ could be observed.

\section{Anxiety ratings}

Similarly to the startle reflex, subjective anxiety ratings also indicated that anxiety and fear were successfully induced, considering the following criteria (see Figure 3B). In the condition $\mathrm{P}$, anxiety ratings should be higher in the presence than in the absence of the visual cue $\left(\mathrm{P}_{\text {cue }}>\mathrm{P}_{\text {nocue }}\right)$, representing the "fear response". Anxiety ratings during the absence of the cue in the condition $\mathrm{U}$ should be higher than during the condition $\mathrm{N}\left(\mathrm{U}_{\text {nocue }}>\mathrm{N}_{\text {nocue }}\right)$, representing the "anxiety response". We found a main effect of condition (P, N and $\mathrm{U})$ $\left(F(1.32,48.84)=88.71, p<0.001, \eta^{2}=0.71\right)$, a main effect of cue (presence or absence of the cue) $(F(1,37)=58,06, p<0.001$, $\left.\eta^{2}=0.61\right)$ and a significant two-way interaction of condition and cue $\left(F(1.62,59.98)=51.36, p<0.001, \eta^{2}=0.58\right)$. Post hoc $t$ tests confirmed both fear $\left(\mathrm{P}_{\text {cue }}>\mathrm{P}_{\text {nocue }} ; t(37)=8.83, p<0.001\right)$ and anxiety $\left(\mathrm{U}_{\text {nocue }}>\mathrm{N}_{\text {nocue }} ; t(37)=9.35, p<0.001\right)$.

\section{Effects of anxiety and fear on pain}

Painfulness of electric stimuli

Although we successfully induced anxiety and fear, results revealed that subjects did not perceive the painfulness of the electric stimuli as different between the anxiety condition (condition $\mathrm{U})(\mathrm{M}=1.86 ; \mathrm{SD}=0.52)$ and the fear condition (condition $\mathrm{P})(\mathrm{M}=1.80 ; \mathrm{SD}=0.49) ;(t(37)=1.71, p=0.1)$.

\section{Effects of anxiety and fear on other ratings}

Additionally, we did not find differences in the perception of the intensity of the electric stimuli between the anxiety condition (condition $\mathrm{U})(\mathrm{M}=2.04 ; \mathrm{SD}=0.42)$ and the fear condition (condition $\mathrm{P})(\mathrm{M}=2.02 ; \mathrm{SD}=0.37) ;(t(37)=0.76, p=0.45)$. Moreover, there were no differences in the perceived threat of the electric stimuli between the anxiety condition (condition $\mathrm{U})(\mathrm{M}=1.89 ; \mathrm{SD}=0.6)$ and the fear condition (condition P) $(\mathrm{M}=1.84 ; \mathrm{SD}=0.54) ;(t(37)=1.41, p=0.17)$.

\section{Influence of anxiety sensitivity (ASI III)}

Direct and moderating influences of ASI III on pain perception

The repeated measures ANOVA yielded a significant main effect of the group level of AS on pain perception $(F(1,36)$ $\left.=2.89, p=0.049, \eta^{2}=0.07\right)$ and a significant interaction between group level of AS and condition (P and $\mathrm{U}$ ) ( $F$ $\left.(1,36)=3.3, p=0.04, \eta^{2}=0.08\right)$. Further, one-tailed $t$-tests showed that H-AS subjects provided higher pain ratings compared to the L-AS subjects in the anxiety condition (condition U) (H-AS: $\mathrm{M}=2.0, \mathrm{SD}=0.52$; L-AS: $\mathrm{M}=1.68$, $\mathrm{SD}=0.48 ; t(36)=1.97, p=0.03$ ). In the fear condition (condition $\mathrm{P}), \mathrm{H}-\mathrm{AS}$ and L-AS subjects did not significantly differ in their pain sensitivity (H-AS: $\mathrm{M}=1.9, \mathrm{SD}=0.5$; LAS: $\mathrm{M}=1.68, \mathrm{SD}=0.45 ; t(36)=1.35, p=0.09)$. Interestingly, H-AS subjects were more pain sensitive in the anxiety condition (condition $\mathrm{U}$ ) than in the fear condition (condition P) (U: $\mathrm{M}=2.0, \mathrm{SD}=0.52 ; \mathrm{P}: \mathrm{M}=1.9, \mathrm{SD}=0.5 ; t(20)$ $=2.5, p=0.01)$. In contrast, L-AS subjects did not differ in their pain perception between the anxiety (condition $\mathrm{U}$ ) 
and the fear condition (condition $\mathrm{P})(\mathrm{U}: \mathrm{M}=1.68, \mathrm{SD}=0.48$; $\mathrm{P}: \mathrm{M}=1.68, \mathrm{SD}=0.45 ; t(16)=-0.174, p=0.43)$. Results show that H-AS subjects were significantly more pain sensitive to the electric stimuli than L-AS subjects, but only in the anxiety condition (condition $\mathrm{U}$ ) and not in the fear condition (condition P), which agrees with our hypotheses (see Figure 4). Thus, AS appeared to moderate the effect of anxiety and fear on pain perception.

Influence of ASI III on other ratings of electrostimulation The repeated measures ANOVA yielded no significant main effect of the group level of AS on the intensity ratings of the electric stimuli $\left(F(1,36)=1.4 ; p=0.24, \eta^{2}=0.04\right.$; condition $\mathrm{U}: \mathrm{H}-$ AS: $\mathrm{M}=2.12, \mathrm{SD}=0.43$; L-AS: $\mathrm{M}=1.93, \mathrm{SD}=0.39$; condition $\mathrm{P}$ : H-AS: $\mathrm{M}=2.06, \mathrm{SD}=0.41$; L-AS: $\mathrm{M}=1.96, \mathrm{SD}=0.32$ ) or the threat ratings of the electric stimuli $(F(1,36)=2.4 ; p=0.13$, $\eta^{2}=0.06$; condition U: H-AS: $\mathrm{M}=2.03, \mathrm{SD}=0.56$; L-AS: $\mathrm{M}=1.71, \mathrm{SD}=0.6$; condition P: H-AS: $\mathrm{M}=1.94, \mathrm{SD}=0.55$; LAS: $\mathrm{M}=1.71, \mathrm{SD}=0.51)$. Moreover, there were no significant interactions between group level of AS and condition ( $\mathrm{P}$ and $\mathrm{U}$ ) for both intensity ratings $\left(F(1,36)=3.68 ; p=0.06, \eta^{2}=0.09\right)$ and threat ratings $\left(F(1,36)=1.85 ; p=0.18, \eta^{2}=0.05\right)$.

\section{Influence of ASI III on startle reflex and anxiety ratings} In order to obtain indicators for the size of fear-potentiated startle/fear response $\left(\mathrm{P}_{\text {cue }}-\mathrm{P}_{\text {nocue }}\right)$ and anxiety potentiated startle/anxiety response $\left(\mathrm{U}_{\text {nocue }}-\mathrm{N}_{\text {nocue }}\right)$, we calculated difference scores between the conditions $\mathrm{P}_{\text {cue }}$ and $\mathrm{P}_{\text {nocue }}$ and between the conditions $U_{\text {nocue }}$ and $N_{\text {nocue }}$ for both ratings and startle amplitudes and compared these scores between H-AS and L-AS by using independent $t$-tests. Results showed that $\mathrm{H}-$

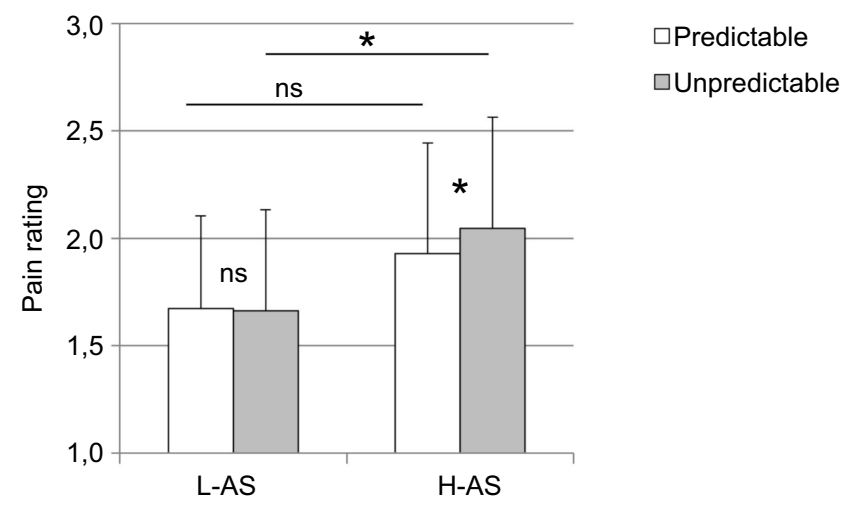

Figure 4 Mean ratings of the painfulness of the electric stimuli in the conditions $P$ and $U$ compared between low anxiety sensitive subjects (L-AS) and high anxiety sensitive subjects (H-AS); * $p<0.05$; bars represent standard deviation; data were square root transformed.

Abbreviation: ns, not significant.
AS subjects and L-AS subjects did not significantly differ in anxiety-potentiated startle (H-AS: diff-score $=0.69, \mathrm{SD}=0.61$; L-AS: diff-score $=1.05, \mathrm{SD}=0.96 ; t(36)=1.43, p=0.16$ ), anxiety response (H-AS: diff-score $=0.93, \mathrm{SD}=0.39$; L-AS: diffscore $=0.67, \mathrm{SD}=0.66 ; t(36)=1.51, p=0.14)$, fear-potentiated startle (H-AS: diff-score $=0.52, \quad \mathrm{SD}=0.71$; L-AS: diffscore $=0.86, \mathrm{SD}=0.71 ; t(36)=1.46, p=0.15)$ or fear responses (H-AS: diff-score $=0.54, \mathrm{SD}=0.22$; L-AS: diff-score $=0.49$, $\mathrm{SD}=0.49 ; t(36)=0.39, p=0.7)$.

\section{Discussion}

In this study, we investigated the effects of anxiety and fear on pain perception with the NPU paradigm. Considering former studies, we assumed that subjects perceive the painfulness of electric stimuli differently in dependence of anxiety or fear.${ }^{11,14}$ Although we successfully induced anxiety and fear as confirmed by startle responses and subjective ratings, we did not observe differences in pain perception between both conditions.

In addition, we examined the moderating influence of AS (as measured by the ASI III) on pain perception in relation to induced anxiety and fear. We assumed that H-AS subjects are more pain sensitive than L-AS subjects. Our results support this hypothesis. H-AS subjects showed enhanced pain sensitivity in contrast to L-AS subjects, but only in the anxiety condition. Interestingly, pain sensitivity differed between the anxiety and the fear condition only in HAS subjects. This means that anxiety enhanced pain perception in HAS subjects, whereas fear did not affect pain sensitivity at all. Previous studies indicated that high AS is generally associated with enhanced pain perception. ${ }^{31,32,35}$ Our results suggest that this association is not perfectly general but rather depends on the emotional predictability of the situation.

However, it is surprising that H-AS subjects did not show significantly enhanced anxiety-responses or enhanced anxietypotentiated startle, although they showed increased pain sensitivity in the anxiety condition. These results appear contradictory and should be clarified in further studies.

We think that with the NPU paradigm, which is originally a tool in anxiety and fear research, we found a possibility for investigating the association between anxiety, fear and pain without the confounding problem of attentional distraction from the pain stimulus by an emotion-inducing second stimulus, which is a major methodological step forward in this domain of pain research. Our results showed that the adaptation of the NPU paradigm as regards the number of electric stimuli, which was necessary to study anxiety and fear effects 
on pain perception, did not negatively affect its efficiency. Specifically, results revealed that anxiety and fear were still successfully induced in our study. Therefore, we think that the adapted NPU paradigm is a useful tool for further studies on the effects of anxiety and fear on pain perception. For example, it might be interesting to use the NPU paradigm to investigate the effects of anxiety and fear on endogenous pain inhibition.

However, it should be mentioned that in conditioning paradigms the general assumption comprises that the strength of the unconditioned stimulus (UCS), which is here both, the anxiety and fear-inducing stimulus and the pain stimulus, is inter-individually stable. Our findings are questioning this prerequisite. It appeared that inter-individual differences in anxiety sensitivity might affect pain sensitivity, which in turn could have influenced the strength of the UCS so that conditioning effects might have varied between subjects. However, this limitation is inherent to all paradigms using conditioning and should not be over-interpreted for the present case.

Our study showed that anxiety but not fear enhanced pain perception in individuals with clinically relevant AS. In other words, the effect of anxiety and fear on pain perception is not ubiquitous but depends on a certain individual vulnerability. Subjects having the predisposition to show exaggerated anxiety sensitivity were more sensitive to pain when they were in uncertain situations. Because of their predisposition to be more responsive to unpredictable threat, H-AS individuals might be especially likely to experience enhanced pain in anxiety-inducing situations. Given that this is a stable response-bias, H-AS persons might be especially prone to develop chronic pain, which is in line with further studies. ${ }^{32,35,46}$

Therefore, in clinical context, it might be worth identifying subjects with high AS to offer them anxiety-sensitivity reducing interventions additionally to the pain treatment. Studies have shown the efficacy of cognitive behavioral therapy to reduce AS. ${ }^{47,48}$ Especially a combination of psychoeducation and interpretation bias modification intervention, more precisely the modification of misinterpretations related to bodily sensations, seems to be successful. ${ }^{48}$ The modification intervention might help high AS subjects to deal with pain in situations with uncertain outcome. This could reduce acute pain and even prevent on the long run the development of chronic pain.

However, offering anxiety-sensitivity reducing interventions only to subjects, which profit from it (subjects with clinically relevant AS), saves resources and money in the health system.

\section{Limitations}

There are also some limitations worth mentioning.

First, in our study electric stimuli were not rated trial-bytrial but retrospectively after each condition. One could possibly argue that this procedure might cause potential memory bias. However, we suppose that it is manageable to retrospectively average three perceptual experiences (three electric stimuli) over six minutes. For note, this method entails the advantage that the induced emotional state was not repeatedly interrupted by asking for ratings after each electric stimulus.

Another noteworthy aspect is that we used electric stimuli, which subjects pre-selected as "unpleasant". Later, subjects were asked to rate the "painfulness" of the electric stimuli. With this pre-selection procedure, we stayed as close as possible to the original NPU paradigm, which enhanced the validity of our experiment as anxiety and fear study. We assume that this change in required perceptual quality is not problematic, given that there is a strong association between unpleasantness and painfulness. ${ }^{49}$

Finally, the low age range of our sample should be noted (subjects only ranged between 20 and 30 years of age). We selected a student sample, thus limiting the age range, because we expected to find sufficiently high AS subjects only in this population, as in our previous studies. Our small sample might also be a limitation, wherefore for future studies, it is recommended to collect data in larger samples with a broader age range in order to enhance external validity.

\section{Conclusion}

Our results have revealed that effects of anxiety on pain perception were moderated by anxiety sensitivity; more precisely, anxiety increases pain in H-AS but not in LAS individuals. This suggests that the effect of anxiety on pain is not ubiquitous but depends on a certain individual emotional vulnerability. Moreover, we found no evidence for pain-modulating effects of fear. The NPU paradigm proved to be a suitable tool to study anxiety and fear effects on pain processing.

\section{Ethics approval and informed consent}

The protocol of the studies will follow the ethical principles for research involving human subjects given in the World Medical Association's Declaration of Helsinki. Informed consent was obtained from all individual participants included in the study. The person in Figure 1 has provided written informed consent for the image to be published. 


\section{Data sharing statement}

Data are shared. 10.6084/m9.figshare.7140053

\section{Acknowledgments}

This study was supported by a research grant from the Deutsche Forschungsgemeinschaft (La 685/13-1).

\section{Disclosure}

The authors report no conflicts of interest in this work.

\section{References}

1. Wiech K, Tracey I. The influence of negative emotions on pain: behavioral effects and neural mechanisms. Neuroimage. 2009;47 (3):987-994. doi:10.1016/j.neuroimage.2009.05.059

2. Bobey MJ, Davidson PO. Psychological factors affecting pain tolerance. J Psychosom Res. 1970;14(4):371-376. doi:10.1016/0022-3999 (70)90003-6

3. Ferreira-Valente MA, Pais-Ribeiro JL, Jensen MP. Associations between psychosocial factors and pain intensity, physical functioning, and psychological functioning in patients with chronic pain. A crosscultural comparison. Clin J Pain. 2014;30(8):713-723. doi:10.1097/ AJP.0000000000000027

4. Macfarlane TV, Kincey J, Worthington HV. The association between psychological factors and oro-facial pain. A community-based study. Eur J Pain. 2002;6(6):427-434. doi:10.1016/S1090-3801(02)00045-9

5. Pincus T, Burton AK, Vogel S, Field AP. A systematic review of psychological factors as predictors of chronicity/disability in prospective cohorts of low back pain. Spine. 2002;27(5):E109-E120. doi:10.1097/00007632-200203010-00017

6. Leeuw M, Goossens MEJB, Linton SJ, Crombez G, Boersma K, Vlaeyen JWS. The fear-avoidance model of musculoskeletal pain: current state of scientific evidence. J Behav Med. 2007;30(1):77-94. doi:10.1007/s10865-006-9085-0

7. Vlaeyen JWS, Linton SJ. Fear-avoidance and its consequences in chronic musculoskeletal pain: a state of the art. Pain. 2000;85 (3):317-332. doi:10.1016/S0304-3959(99)00242-0

8. Weissman-Fogel I, Sprecher E, Pud D. Effects of catastrophizing on pain perception and pain modulation. Exp Brain Res. 2008;186 (1):79-85. doi:10.1007/s00221-007-1206-7

9. Rainville P, Bao QVH, Chretien P. Pain-related emotions modulate experimental pain perception and autonomic responses. Pain. 2005;118(3):306-318. doi:10.1016/j.pain.2005.08.022

10. Malow RM. The effects of induced anxiety on pain perception: A signal detection analysis. Pain. 1981;11(3):397-405. doi:10.1016/ 0304-3959(81)90639-4

11. Rhudy JL, Meagher MW. Fear and anxiety: divergent effects on human pain thresholds. Pain. 2000;84:65-75. doi:10.1016/S03043959(99)00183-9

12. Tang J, Gibson SJ. A psychophysical evaluation of the relationship between trait anxiety, pain perception, and induced state anxiety. $J$ Pain. 2005;6(9):612-619. doi:10.1016/j.jpain.2005.03.009

13. Rhudy JL, Meagher MW. Negative affect. Effects on an evaluative measure of human pain. Pain. 2003;104(3):617-626. doi:10.1016/ S0304-3959(03)00119-2

14. Al Absi M, Rokke PD. Can anxiety help us tolerate pain? Pain. 1991;46:43-51. doi:10.1016/0304-3959(91)90032-S

15. Davis M. Are different parts of the extended amygdala involved in fear versus anxiety? Biol Psychiatry. 1998;44(12):1239-1247. doi:10.1016/S0006-3223(98)00288-1
16. Wied MD, Verbaten MN. Affective pictures processing, attention, and pain tolerance. Pain. 2001;90(1):163-172. doi:10.1016/S03043959(00)00400-0

17. Davis M, Walker DL, Miles L, Grillon C. Phasic vs sustained fear in rats and humans. Role of the extended amygdala in fear vs anxiety. Neuropsychopharmacology. 2010;35(1):105-135. doi:10.1038/ npp.2009.109

18. Rhudy JL, Meagher MW. The role of emotion in pain modulation. Curr Opin Psychiatry. 2001;14(3):241-245. doi:10.1097/00001504200105000-00012

19. Crombez G, Eccleston C, van Den Broeck A, Goubert L, van Houdenhove B. Hypervigilance to pain in fibromyalgia. Clin $J$ Pain. 2004;20(2):98-102. doi:10.1097/00002508-200403000-00006

20. Lautenbacher S, Huber C, Kunz M, et al. Hypervigilance as predictor of postoperative acute pain. Its predictive potency compared with experimental pain sensitivity, cortisol reactivity, and affective state. Clin $J$ Pain. 2009;25(2):92-100. doi:10.1097/AJP.0b013e3181850dce

21. Meagher MW, Arnau RC, Rhudy JL. Pain and emotion. Effects of affective picture modulation. Psychosom Med. 2001;63(1):79-90. doi:10.1097/00006842-200101000-00010

22. Schmitz A, Grillon C. Assessing fear and anxiety in humans using the threat of predictable and unpredictable aversive events (the NPU-threat test). Nat Protoc. 2012;7(3):527-532. doi:10.1038/nprot.2012.001

23. Grillon C, Davis M. Fear-potentiated startle conditioning in humans. Explicit and contextual cue conditioning following paired versus unpaired training. Psychophysiology. 1997;34(4):451-458. doi:10.1111/j.14698986.1997.tb02389.x

24. Brosch T, Scherer KR, Grandjean D, Sander D. The impact of emotion on perception, attention, memory, and decision-making. Swiss Med Wkly. 2013;143:w13786. doi:10.4414/smw.2013.13786

25. Yiend J. The effects of emotion on attention: A review of attentional processing of emotional information. Cogn Emot. 2010;24(1):3-47. doi:10.1080/02699930903205698

26. Sloan P, Hollins M. Attention and pain: are auditory distractors special? Exp Brain Res. 2017;235(5):1593-1602. doi:10.1007/ s00221-017-4903-x

27. Kenntner-Mabiala R, Weyers P, Pauli P. Independent effects of emotion and attention on sensory and affective pain perception. Cogn Emot. 2007;21(8):1615-1629. doi:10.1080/02699930701252249

28. Reiss S, Peterson RA, Gursky DM, McNally RJ. Anxiety sensitivity, anxiety frequency and the prediction of fearfulness. Behav Res Ther. 1986;24(1):1-8. doi:10.1016/0005-7967(86)90143-9

29. Carleton RN, Sharpe D, Asmundson GJG. Anxiety sensitivity and intolerance of uncertainty. Requisites of the fundamental fears? Behav Res Ther. 2007;45(10):2307-2316. doi:10.1016/j.brat.2007.04.006

30. Nelson BD, Hodges A, Hajcak G, Shankman SA. Anxiety sensitivity and the anticipation of predictable and unpredictable threat. Evidence from the startle response and event-related potentials. $J$ Anxiety Disord. 2015;33:62-71. doi:10.1016/j.janxdis.2015.05.003

31. Stewart SH, Asmundson GJG. Anxiety sensitivity and its impact on pain experiences and conditions. A state of the art. Cogn Behav Ther. 2006;35(4):185-188. doi:10.1080/16506070601090457

32. Asmundson GJG. Anxiety sensitivity and chronic pain. Empirical findings, clinical implications, and future directions. In: Taylor S, editor. Anxiety Sensitivity. Theory, Research, and Treatment of the Fear of Anxiety. Mahwah N.J: L. Erlbaum Associates; 1999:269-286.

33. Tegethoff M, Belardi A, Stalujanis E, Meinlschmidt G. Comorbidity of mental disorders and chronic pain: chronology of onset in adolescents of a national representative cohort. J Pain. 2015;16(10):10541064. doi:10.1016/j.jpain.2015.06.009

34. Schmidt NB, Cook JH. Effects of anxiety sensitivity on anxiety and pain during a cold pressor challenge in patients with panic disorder. Behav Res Ther. 1999;37(4):313-323. doi:10.1016/S0005-7967(98) 00139-9 
35. Keogh E, Cochrane M. Anxiety sensitivity, cognitive biases, and the experience of pain. J Pain. 2002;3(4):320-329. doi:10.1054/ jpai.2002.125182

36. Keogh E, Asmundson GJ. Negative affectivity, catastrophizing, and anxiety sensitivity. In Asmundson GJ, Vlaeyen JWS, Combez G, editors. Understanding and Treating Fear of Pain. Oxford, England: Oxford University Press; 2004:.99-115.

37. Taylor S, Zvolensky MJ, Cox BJ, et al. Robust dimensions of anxiety sensitivity: development and initial validation of the Anxiety Sensitivity Index-3. Psychol Assess. 2007;19(2):176-188. doi:10.1037/10403590.19.2.176

38. Kemper CJ, Ziegler M, Taylor S. Überprüfung der psychometrischen Qualität der deutschen Version des Angstsensitivitätsindex-3. Diagnostica. 2009;55(4):223-233. doi:10.1026/0012-1924.55.4.223

39. Allan NP, Raines AM, Capron DW, Norr AM, Zvolensky MJ, Schmid NB. Identification of anxiety sensitivity classes and clinical cut-scores in a sample of adult smokers. Results from a factor mixture model. J Anxiety Disord. 2014;28(7):696-703. doi:10.1016/j.janxdis.2014.07.006

40. Lang PJ, Bradley MM, Cuthbert BN. Emotion, attention, and the startle reflex. Psychol Rev. 1990;97(3):377-395. doi:10.1037/0033295X.97.3.377

41. Grillon C, Baas J. A review of the modulation of the startle reflex by affective states and its application in psychiatry. Clin Neurophysiol. 2003;114(9):1557-1579. doi:10.1016/S1388-2457(03)00202-5

42. Blumenthal TD, Cuthbert BN, Filion DL, Hackley S, Lipp OV, van Boxtel A. Committee report: guidelines for human startle eyeblink electromyographic studies. Psychophysiology. 2005;42(1):1-15. doi:10.1111/j.1469-8986.2005.00271.x
43. Horn-Hofmann C, Priebe JA, Schaller J, Görlitz R, Lautenbacher S. Lack of predictive power of trait fear and anxiety for conditioned pain modulation (CPM). Exp Brain Res. 2016;234:3649-3658. doi:10.1007/s00221-016-4763-9

44. Horn C, Blischke Y, Kunz M, Lautenbacher S. Does pain necessarily have an affective component? Negative evidence from blink reflex experiments. Pain Res Manag. 2012;17(1):15-24.

45. Horn-Hofmann C, Lautenbacher S. Modulation of the startle reflex by heat pain: does threat play a role? Eur J Pain. 2015;19(2):216224. doi:10.1002/ejp.539

46. Asmundson GJG, Wright KD, Hadjistavropoulos HD. Anxiety sensitivity and disabling chronic health conditions. State of the art and future directions. Scand J Behav Ther. 2000;29(3-4):100-117. doi:10.1080/028457100300049719

47. Smits JA, Berry AC, Tart CD, Powers MB. The efficacy of cognitive behavioral interventions for reducing anxiety sensitivity: a meta analytic review. Behav Res Ther. 2008;46(9):10471054. doi:10.101 6/j.brat.2008.06.01048

48. Capron DW, Norr AM, Allan NP, Schmidt NB. Combined "topdown" and "bottom-up" intervention for anxiety sensitivity: pilot randomized trial testing the additive effect of interpretation bias modification. J Psychiatr Res. 2017;85:75-82. doi:10.1016/j. jpsychires.2016.11.003

49. Price D, Harkins S. The affective-motivational dimension of pain A two-stage model. APS J. 1992;1(4):229-239. doi:10.1016/1058-9139 (92)90054-G
Journal of Pain Research

\section{Publish your work in this journal}

The Journal of Pain Research is an international, peer reviewed, open access, online journal that welcomes laboratory and clinical findings in the fields of pain research and the prevention and management of pain. Original research, reviews, symposium reports, hypothesis formation and commentaries are all considered for publication. The manuscript management system is completely online and includes a very quick and fair peer-review system, which is all easy to use. Visit http:// www.dovepress.com/testimonials.php to read real quotes from published authors. 